\title{
Carrier sense range effect on multipath routing performances in Wireless Sensor Networks
}

\author{
I. Bennis* ${ }^{*}$, H. Fouchal ${ }^{\ddagger}$, O. Zytoune ${ }^{* \S}$, D. Aboutajdine* \\ *LRIT, unité associée au CNRST (URAC29), Université Mohammed V - Agdal, Rabat, Marocco \\ ‡Université de Reims Champagne-Ardenne, France \\ $\S$ Université Ibn Tofail, Kénitra, Maroc \\ Email: i.bennis@fsr.um5a.ma, hacene.fouchal@univ-reims.fr, zytoune@univ-ibntofail.ac.ma, aboutaj@fsr.ac.ma
}

\begin{abstract}
In the recent years, Wireless Sensor Networks (WSNs) have become a great field of interest for scientific community. This kind of network provides a panoply of applications in different areas of human life. However WSNs must ensure the quality of service $(\mathrm{Q} O S)$ to give the requested performance for the final user. Among different issues presented in the literature to provide high QoS, multipath routing is commonly used. But such a solution could be not enough efficient if the multipath routing design does not consider the phenomena of interference. Indeed the constructed paths can have an interference zone, mainly a shared carrier sense range. In this paper we show by analytical and experimental results, that using multipath routing can never overshoot the performance of single path when the interferences are not taken into account. Also, we show how the carrier sense range can influence the network performance.
\end{abstract}

Index Terms-WSN, Carrier sense range, Interference, Multipath routing, QoS.

\section{INTRODUCTION}

$\mathbf{N}$ OWADAYS Wireless Sensor Networks (WSNs) attract more and more researchers as being an interdisciplinary research of interest. This kind of networks offers countless applications like precise agriculture, system monitoring and many others. One of the most challenging issue in these networks is that the routing techniques must satisfy some QoS metrics. Especially in the case of multimedia applications that require the best performance in terms of energy consumption, delay, jitter, reliability and bandwidth.

There are many solutions that can be included in the protocol design to ensure QoS requirements as using multipath routing. This technique consists of giving a source node the possibility to use any of several paths to reach a particular destination at any time. According to [1], [2], the use of a multipath routing has many benefits such as aggregation of bandwidth by splitting data to the same destination into multiple streams. Also, the use of the multipath principle can reduce the end to end delay in case of route failure because there is no need to restart a new path discovery process. In addition, multipath routing has the ability to improve the reliability of the transmitted information by sending multiple copies of the same data on multiple paths, which increases the accuracy. Another interesting benefit of multipath techniques is the load balancing which allows a better use of available network resources in order to reduce traffic congestion.
To enhance the multipath routing scheme design, many works [3], [4] use the notion of disjoint paths. This notion reflects the independence of paths in terms of shared resources. The higher is the degree of independence, the more the multipath strategy promotes an adaptive use of the network resources. With such a feature, the multipath solution can avoid congestion by balancing the load among the multiple disjoint paths. In the literature, several techniques based on the degree of disjoint paths are used to classify the set of paths between a source node and a destination node. Among them we cite Link-disjoint, Node-disjoint, Maximally-disjoint and Radio-disjoint Multipath [1].

However multipath solution hides a serious drawback. In fact since a single channel is used in the wireless network, the sensors nodes share the medium of communication. And due to the broadcast nature of radio communication the level of interference is more pronounced [5], [6]. Also, when several paths are used simultaneously, even if the node disjoint priority is satisfied, it remains a significant risk of collisions that results in high packet loss rate. So the concurrent use of multiple paths constructed from source to destination results in intensive inter-path interference [7]. Therefore performances will decrease seriously. Authors in [8] concluded that transmitting data over multiple paths is not a synonym of result improvement unless the effects of the wireless communications are taken into account. This phenomena is also known as the route-coupling problem [9]. It occurs when simultaneous communications through multiple paths are ongoing, and these paths are located physically close enough in order to interfere with each other.

Another aspect that must be considered, is the effect of the carrier sense range on communication performances in sensor networks, mainly in routing protocol. Indeed, while the carrier sense range is usually more larger than the transmission range, there is more chance that interferences occur in this range, especially in the case of high density. We mean by carrier sense range effect the fact that a node cannot transmit as an other node in its carrier sense range is already in a transmitting phase.

In this work we study how the carrier sense range can influence the communication in a sensor network. We show by analytical and experimental results that using multipath routing can never overshoot the performances of a single path when 
the carrier sense range effect is not considered in the routing design.

The remainder of this paper is organized as follows: in section II, some routing protocols with the aim of reducing the interference problem in the multipath case are presented. In section III, we describe by analytical model the effect of both wireless interferences and carrier sensing. Simulation results are shown in section IV. Finally in section V, we draw the conclusion and give some perspectives.

\section{RELATED WORK}

In the literature, there exists some solutions that aim to reduce the effects of interference such as directed antennas [10]. The authors try to find a zone-disjoint multipath to avoid collisions between paths. Another solution consists of using multi-channel transmission [11]. But these both methods cannot be easily used due to the resource-constrained propriety of the WSNs. An alternative technique is to calculate the degree of independence between a set of paths using the correlation factor or the coupling metrics. The correlation factor between two node-disjoint paths is defined as the total number of shared links of the paths [5]. It represents the chances that the transmission along the different paths could interfere with each other in a shared channel. The coupling between two paths ( $\mathrm{Pl}$ and $\mathrm{P} 2$ ) is defined as the average number of nodes that are unable to receive data along P2 when a single node in P1 is transmitting [9]. The more the path has lower correlation factor or coupling effect, the more suitable is for multipath construction allowing better performances.

In the following, we will review some studies about routing protocols that aim reducing the interference problem in the multipath case.

- Energy Efficient Collision Aware Multipath Routing for WSN (EECA) [12]:

The EECA is an on-demand routing protocol that constructs multiple paths using request/reply cycles. This protocol has two aims:

- Reducing the flooding of route request messages by restricting it to the neighbors of nodes iteratively added to the route being discovered.

- Saving energy by adjusting power needed to transmit the data and the control messages and so reducing the potential collision area of each node.

The author makes assumption that each node can adjust the radio transmit power to vary its communication range from 0 to a specific transmit range. The EECA algorithm attempts to find two collision-free routes using the node position information. The source starts by checking if there are two groups in its neighbor list satisfying the following three conditions: 1) all these nodes are close to the destination; 2) The nodes of the two groups are opposite and separated by the source-destination line; 3 ) each node is distanced more than $\mathrm{R} / 2$ from the source destination line.

However such restrictions limit the chance to find two paths far away from each other. So many nodes for the first constructed path remain in the carrier sense range of other nodes for the second path.

- Interference-Minimized Multipath Routing with Congestion Control in Wireless Sensor Network for High-Rate Streaming (I2MR) [13]:

This protocol tries to increase the throughput by discovering zone-disjoint paths and adopting the load balancing scheme, while requiring minimal geographic information to reduce overheads. Localization support is only required at the source nodes, which are the most powerful sensor nodes equipped with Electro-Optic devices. The basic idea is to mark-out the interference zone of the nodes of the first path after it has been discovered. Then subsequent paths cannot be discovered within this interference zone. I2MR tries to construct zone-disjoint paths and distributes network traffic over the discovered paths by assuming a special network structure and the availability of particular hardware components. In I2MR, the source node tries to find three paths, but uses the two first paths for data transmission and keeps the third one as a backup path. However, this work needs a special network structure and particular hardware components making this protocol not applicable to all types of sensors. In addition, due to the high complexity of the introduced zonemarking mechanism, and the different type of packet control, the generated overhead is more pronounced.

- Maximally radio-disjoint multipath routing for wireless multimedia sensor networks (MR2) [14]

The main objective for the MR2 protocol is to provide the required bandwidth for multimedia applications through non-interfering paths. MR2 utilizes an adaptive incremental technique to construct minimum-interfering paths. To do so, only one path is built for a given session. Additional paths are built when required, typically in case of congestion or bandwidth shortage. Interference awareness and energy saving are achieved by switching a subset of sensor nodes in a passive state in which they do not take part in the routing process. The passive state is represented by switching the sensor node to a sleep or an idle mode. Thus enabling increasing the network lifetime. However, MR2 is only suitable for query-driven applications. Also, the utilized flooding strategy for constructing non-interfering paths implies a high control overhead. And as the bepassive message is received only by the neighbors of each node forming the path, the constructed paths are spaced by a distance approximately equal to the transmission range. So the carrier sense range effect is not considered.

So for all discussed works the constructed paths are spaced by a distance equal to the transmission range or at most the interference range. Thus the carrier sense range has never been treated.

\section{AnAlytical MODEL}

In this section, we will give a model for a wireless sensor network as a connectivity graph. After that by using the 
protocol model of interference and the physical model of interference we will describe the effects of wireless interferences and of carrier sensing. And finally by a conflict graph we will present the different relations between the wireless links in the network.

\section{A. Connectivity graph}

We consider a wireless network with $\mathrm{N}$ nodes randomly located on a plane space. We denote our network by $G(V, E)$ where $\mathrm{V}$ is the vertex that represents a set of $\mathrm{N}$ nodes and $\mathrm{E}$ is the edge that represents set of directed links connecting the nodes in $\mathrm{V}$. Let $n_{k}$ where $1 \leq k \leq N$ denote the nodes in $V$, and $l_{i j}$ and $d_{i j}$ denote respectively the directed link and the distance between nodes $n_{i}$ and $n_{j}$ with $i, j \in[1, N]$. The notation $l_{i j}$ means also that the node $n_{i}$ sends packet to node $n_{j}$, so $l_{i j}$ has not the same meaning than $l_{j i}$. Each node, $n_{k} \in V$, is equipped with a radio having three levels of range centred at the node $n_{k}$ :

- The transmission range $R_{T r}(k)$, is the range where a successful communication can be achieved.

- The interference range $R_{I}(k)$, is the range where every node that attempts to start a communication will cause collision at node $n_{k}$ when it receives packet.

- The carrier sense range $R_{C S}(k)$, is the range where every node that attempts to start communication will prevent node $n_{k}$ to transmit.

The relationship between the three ranges is $R_{T r}(i)<$ $R_{I}(i)<R_{C S}(i)$. We notice that the nodes are homogeneous, so each node has the same ranges than others. In our study we consider the case of a single wireless channel.

According to the protocol model of interference [15], there is a successful transmission between nodes $n_{i}$ and $n_{j}$ if the following conditions are satisfied:

- $d_{i j} \leq R_{T r}(i)$.

- no transmitting nodes in the potential zone of interference of node $n_{j}$.

- no nodes in the $R_{C S}(i)$ that sends packet concurrently with the node $n_{i}$.

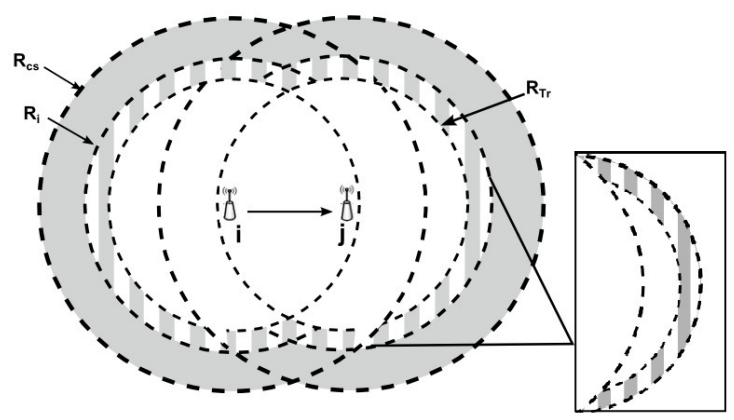

Fig. 1: Potential zone of interference

The potential zone of interference is shown in Fig.1. It is delimited by the intersection of carrier sense range of transmitter (node $n_{i}$ ) and the interference range and transmission range of the receiver (node $n_{j}$ ). The area of this zone depends on the distance between the transmitter and the receiver.

If we consider the physical model of interferences [15] a successful transmission arises only if the signal strength of the received frame at node $n_{j}$ is stronger than RxThresh_. Otherwise if signal strength is less than RxThresh_but greater than CSThresh, the receiver will not be able to decode correctly the signal and the channel is considered as busy. In the case when multi-frames are received simultaneously by node $n_{j}$, it calculates the ratio of the strongest frame signal strength to the signal strength sum of other frames. If it is larger than CPThresh_, the frame will be received correctly and other frames are ignored. Otherwise, all frames are discarded. This case represents the interference that can be occurred at the receiver. The three values RxThresh_, CSThresh_ and CPThresh_are specific thresholds for the wireless node. NS-2 [16] uses the above description to simulate the reception of signal.

\section{B. Conflict graph}

In order to show which wireless links interfere with each other in the network we consider a conflict graph $G^{\prime}\left(V^{\prime}, E^{\prime}\right)$ where $V^{\prime}$ is the vertex that represents each link in the connectivity graph $G$, and $E^{\prime}$ is the edge which represents the set of all possible relations between the vertices in $V^{\prime}$.

Based on the protocol interference model described above, an edge can be drawn between two vertices $l_{i j}$ and $l_{p q}$ in $G^{\prime}$ if the links $l_{i j}$ and $l_{p q}$ may be active simultaneously. Such a condition is achieved if one of the two following conditions are true:

- $i \in R_{I}(q)$ or $p \in R_{I}(j)$.

- $i \in R_{C S}(p)$ or $p \in R_{C S}(i)$.

\section{Multipath case}

Our aim in this subsection is to show how the multipath routing reacts under the three ranges defined above. Namely how each node in each path will interact with other nodes in the other paths. Let have two paths from the source $S$ and the sink $(a-b-c-d)$ and $(e-f-g)$ as mentioned in Fig.2.

In the first path the node $b$ can only forward its data if the nodes $a$ and $c$ are not in the transmitting phase. The same observation can be expected for the node $c$ and the nodes $b$ and $d$. We notice that the transmission is done by a broadcast way due to the nature of radio device.

At the second path, if the node $g$ in $R_{I}(c)$ starts transmission to the sink (if $d$ and $c$ do not transmit), then the receiving data at the node $c$ from the node $b$ will be disrupted. Also, if the node $f$ starts forwarding, it will be in a competition with nodes $b$ and $c$ at the same time as it is located in the carrier sense range of the two nodes; which will cause delays and packet losses. If the node $a$ sends packets to node $b$, this one will be able to successfully receive the packets without any potential interference caused by the node $f$.

\section{Simulation AND EXPERIMENTAL RESUltS}

The aim of this simulation is to show how the same routing protocol performs in single path case and in the multipath 


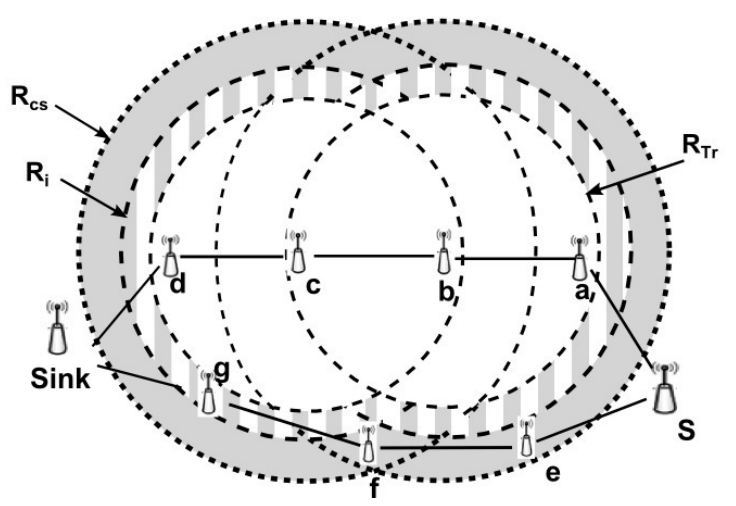

Fig. 2: Multipath study under the three ranges

case without considering the multipath effect. Also, we investigate how the carrier sense range can influence the routing results. We choose as routing protocol for this study our implementation [17] of the Two Phase geographical Greedy Forwarding (TPGF) as it has been one of the first protocol which introduces the concept of multi-paths in the field of WMSNs [18]. This algorithm focuses on the exploration and the establishment of a maximum number of best disjoint routes in terms of end-to-end delay. We evaluate the performance of the studied protocol under the delay metrics to measure the average end-to-end delay of successful received packets. And under the Packet Delivery Ratio (PDR) metrics to calculate the ratio between the number of correctly received packets at the destination and the number of packets sent by the source.

\section{Working environment}

Here we will describe our studied scenario. Each simulation scenario is presented as follows: $\mathbf{X}$ nodes are randomly located in an area of $1500 * 1500 \mathrm{~m}^{2}$, where:

\section{$\mathrm{X}=\llbracket 100 ; 150 ; 200 ; 250 ; 300 \rrbracket$}

Data traffic is generated by a randomly source in the network to a sink. This one is located in the center of our experimentation area and has the last ID. The source node generates a constant bit rate sources (CBR) traffic with $\mathbf{Y}$ packets per second, where:

\section{$\mathrm{Y}=\llbracket 16 ; 32 ; 64 ; 128 ; 256 \rrbracket$}

The data packet size is 1000 bytes. The duration of communication is 40 seconds, an no mobility is supported in this scenario. For every value of $\mathbf{X}$ and $\mathbf{Y} 50$ scenarios are generated and the average value of the results are calculated. Due to the lack of space we represent only the results in case of 64 packets per second.

Table I summarizes the parameters used for simulation.

\section{Result analysis}

From Fig. 3, we notice that for both multipath and single path cases, the average delay decreases as the network density grows, it is quite normal as we have only one source for each
TABLE I: Main configuration parametres

\begin{tabular}{|l|c|}
\hline Parametres & value \\
\hline link layer & LL \\
MAC layer & IEEE 802.11 \\
radio propagation & two ray ground \\
interface queue & PriQueue \\
ifqlen & 50 \\
antenna & omni-antenna \\
CPThreshold (Watt) & 10 \\
CSThreshold (Watt) & $1.559 * 10^{-11}$ \\
RXThreshold (Watt) & $3.652 * 10^{-11}$ \\
Pt (Watt) & 0.2818 \\
\hline
\end{tabular}

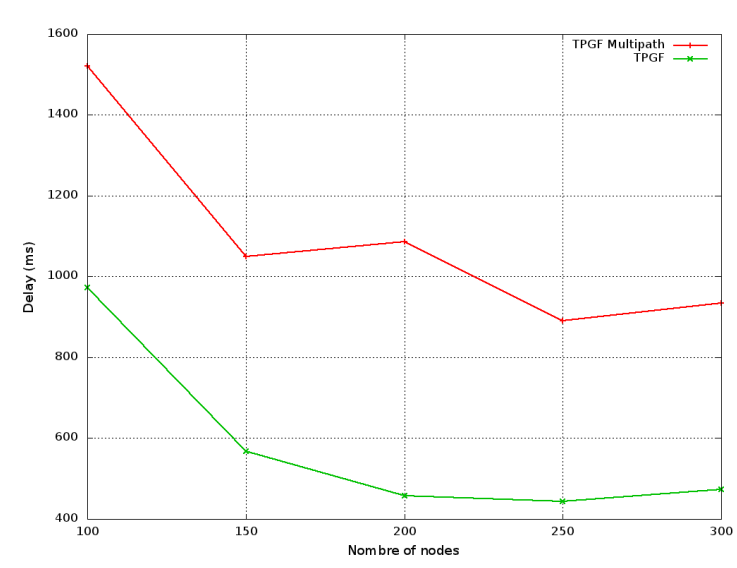

Fig. 3: Average delay vs network size

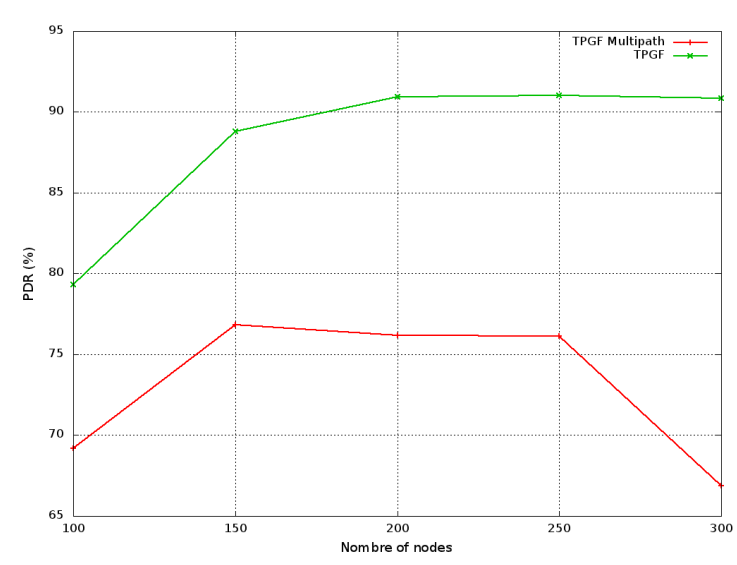

Fig. 4: Average PDR vs network size

scenario. Also, the main observation that Fig. 3 shows is the fact that the single path has the best average delay against the multipath case. The gain is approximately equal to $70 \%$.

This result is expectable since the TPGF protocol does not take into account the interference problem. For Fig. 4 we can see also that the single path has the best average PDR against the multipath case. The gain is approximately equal to $20 \%$.

This part of results confirms that using multipath routing 
without taking into consideration interference and the carriers sense range effects makes the multipath solution less profitable than a single path one.

In order to show how the effect of the carrier sense range can influence performances of routing protocols, we discuss the following scenario. First of all, we modify the TPGF protocol in order to have two version. The first one represents the case where the different paths constructed from the source to the sink have at least a distance from each other equal to the transmission range. We denote this version by $R X$-avoid. The second version represents the case where the paths constructed from the source to the sink have at least a distance from each other equal to carrier sense range. We denote this version by CS-avoid. In this simulations, we have used a grid with 100 nodes spaced by a distance equal to $200 \mathrm{~m}$. The area of the grid is $4000 * 2500 \mathrm{~m}^{2}$. The source node is selected randomly and generatesa constant bit rate (CBR) traffic with $\mathbf{Y}$ packets per second, where:

$$
\mathrm{Y}=\llbracket 16 ; 32 ; 64 ; 80 ; 128 ; 256 \rrbracket
$$

In this simulation we simply use two paths. We notice also that if there is no way to find the second path with the desired condition, we use only the first path. We repeat the simulations several times and we measure the average of delay sand PDRs.

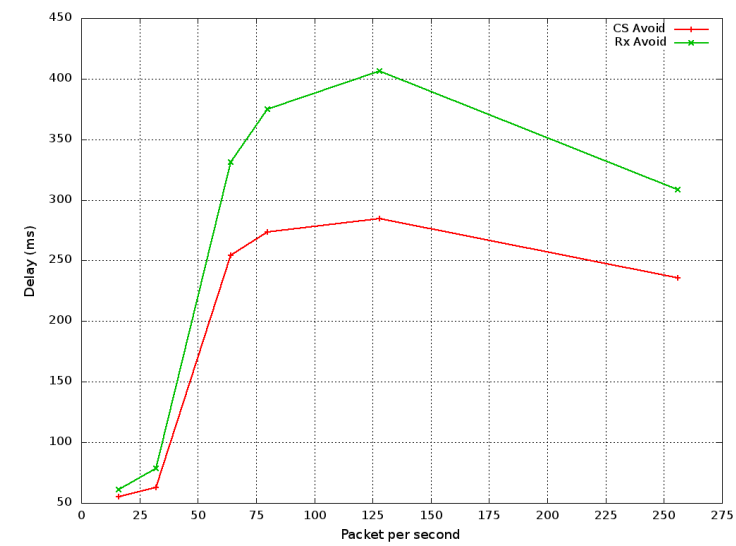

Fig. 5: Average delay vs Rate

From Fig. 5, we notice that for both $C S$-avoid and $R X$-avoid case the average delay increases as the rate grows espacialy from 16 to 128 packet per second. It is quite expected as more the rate is higher more the queue of intermediate nodes is filled. So each packet takes more time to reach destination. But the main observation that we can make from Fig. 5 is the fact that the CS-avoid case has the best average delay against the $R X$-avoid case.

As discussed in section III, the carrier sense range effect occurs when a node cannot transmit as an other node in its carrier sense range is already in transmitting phase. This is exactly the case here, in fact more the number of packets per second increases, more the need for channel access is higher. Therefore the nodes of the two paths deprive mutually the channel access since there is a competition between them. For

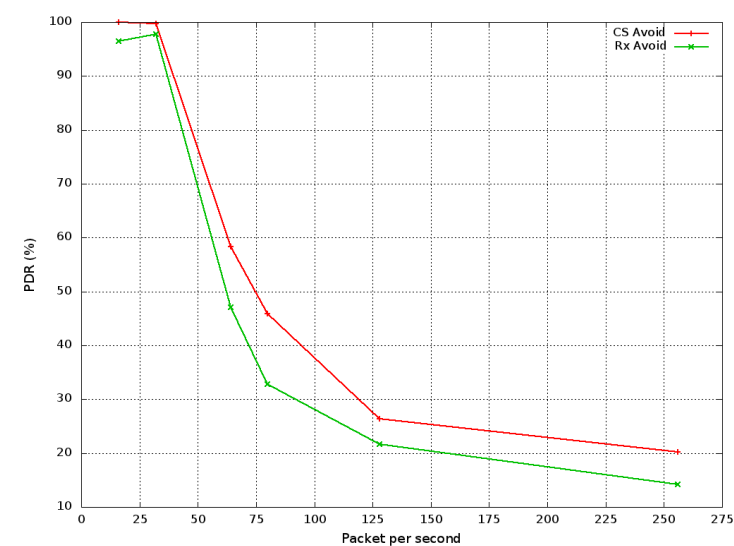

Fig. 6: Average PDR vs Rate

Fig. 6, we can see also that the multipath with the CS-avoid has the best average PDR against the $R X$-avoid case.

\section{CONCLUSION AND Future Work}

Using the multipath routing in the WSN has many benefits such as aggregation of bandwidth, reducing the end to end delay, improving reliability. However, using the multipath routing scheme without considering the effect of the carrier sense range decreases the network performances instead of enhancing it.

We have presented in this paper how the carrier sense range can influence the performance of communication in wireless sensor networks. We show by analytical and experimental results that using multipath techniques can never overshoot the performances of a single path solution unless the carrier sense range effect is considered in the routing design.

We have performed a large number of simulations. Their results prove that multipath with the $C S$-avoid outperforms the multipath with $R X$-avoid for both metrics: delay and PDR. As a future work, we intend to achieve much more simulations for large scale situations and will consider mobility feature. In addition, we need to experiment this protocol with realistic streams which model multimedia ones.

\section{ACKNOWLEDGMENT}

This work is partially supported by "Projet de coopération Maroco-Française : Contribution à l'optimisation de la qualité de service dans les réseaux de capteurs sans fil : Application à la supervision agricole 2013-2014".

This work is partially supported by the "Projet SCOOP@F : Système de Coopération Pilote" funded by the EC 2014-2015.

\section{REFERENCES}

[1] V. D. Mytri Jayashree A, G. S. Biradar. Review of multipath routing protocols in wireless multimedia sensor network -a survey. International Journal of Scientific and Engineering Research, 3(9):174-182, 2012.

[2] H. Zafar, D. Harle, I. Andonovic, and Y. Khawaja. Performance evaluation of shortest multipath source routing scheme. Communications, IET, 3(5):700-713, 2009. 
[3] Zhongdong Wang and Qiushuang Wang. The research of a multiple disjoint paths routing protocol for ad hoc sensor networks. Energy Procedia, 17, Part A(0):499 - 505, 2012. International Conference on Future Electrical Power and Energy System.

[4] U.B. Mahadevaswamy and M.N. Shanmukhaswamy. Delay aware and load balanced multi-path routing in wireless sensor networks. International Journal of Wireless Information Networks, 19(3):278-285, 2012.

[5] Kui $\mathrm{Wu}$ and J. Harms. Performance study of a multipath routing method for wireless mobile ad hoc networks. In Modeling, Analysis and Simulation of Computer and Telecommunication Systems, 2001. Proceedings. Ninth International Symposium on, pages 99-107, 2001.

6] Marjan Radi, Behnam Dezfouli, Kamalrulnizam Abu Bakar, Shukor Ab Razak, and Mohammad Ali Nematbakhsh. Interference-aware multipath routing protocol for qos improvement in event-driven wireless sensor networks. Tsinghua Science \& Technology, 16(5):475 - 490, 2011.

[7] Marjan Radi, Behnam Dezfouli, Kamalrulnizam Abu Bakar, and Malrey Lee. Multipath routing in wireless sensor networks: Survey and research challenges. Sensors, 12(1):650-685, 2012

[8] E.P.C. Jones, M. Karsten, and P.A.S. Ward. Multipath load balancing in multi-hop wireless networks. In Wireless And Mobile Computing, Networking And Communications, 2005. (WiMob'2005), IEEE International Conference on, volume 2, pages 158-166 Vol. 2, Aug 2005.

[9] M.R. Pearlman, Z.J. Haas, P. Sholander, and S.S. Tabrizi. On the impact of alternate path routing for load balancing in mobile ad hoc networks. In Mobile and Ad Hoc Networking and Computing, 2000. MobiHOC. 2000 First Annual Workshop on, pages 3-10, 2000

[10] Dola Saha, Siuli Roy, Somprakash Bandyopadhyay, Somprakash B, Tetsuro Ueda, and Shinsuke Tanaka. An adaptive framework for multipath routing via maximally zone-disjoint shortest paths in ad hoc wireless networks with directional antenna. In In IEEE Global Telecommunications Conference, pages 226-230, 2003.

[11] Wai-Hong Tarn and Yu-Chee Tseng. Joint multi-channel link layer and multi-path routing design for wireless mesh networks. In INFOCOM 2007. 26th IEEE International Conference on Computer Communications. IEEE, pages 2081-2089, May 2007.

[12] Zijian Wang, E. Bulut, and B.K. Szymanski. Energy efficient collision aware multipath routing for wireless sensor networks. In Communications, 2009. ICC '09. IEEE International Conference on, pages 1-5, June 2009.

[13] Jenn-Yue Teo, Yajun Ha, and Chen-Khong Tham. Interferenceminimized multipath routing with congestion control in wireless sensor network for high-rate streaming. Mobile Computing, IEEE Transactions on, 7(9):1124-1137, Sept 2008

[14] Moufida Maimour. Maximally radio-disjoint multipath routing for wireless multimedia sensor networks. In Proceedings of the 4th ACM Workshop on Wireless Multimedia Networking and Performance Modeling, WMuNeP '08, pages 26-31, New York, NY, USA, 2008. ACM.

[15] Kamal Jain, Jitendra Padhye, VenkataN. Padmanabhan, and Lili Qiu. Impact of interference on multi-hop wireless network performance. Wireless Networks, 11(4):471-487, 2005.

[16] VINT. The network simulator ns-2.34, 2012.

[17] I Bennis, H. Fouchal, O. Zytoune, and D. Aboutajdine. An evaluation of the tpgf protocol implementation over ns-2. In Communications (ICC) 2014 IEEE International Conference on, pages 428-433, June 2014

[18] Lei Shu, Yan Zhang, LaurenceT. Yang, Yu Wang, Manfred Hauswirth, and Naixue Xiong. Tpgf: geographic routing in wireless multimedia sensor networks. Telecommunication Systems, 44(1-2):79-95, 2010. 